\title{
Itinerant density wave instabilities at classical and quantum critical points
}

Yejun Feng ${ }^{1,2}$, Jasper van Wezel ${ }^{3}$, Jiyang Wang ${ }^{2}$, Felix Flicker ${ }^{4}$, D. M. Silevitch ${ }^{2}$, P. B. Littlewood $^{2,5}$, T. F. Rosenbaum ${ }^{2,6}$

${ }^{1}$ The Advanced Photon Source, Argonne National Laboratory, Argonne, Illinois 60649, USA

${ }^{2}$ The James Franck Institute and Department of Physics, The University of Chicago, Chicago, Illinois 60637, USA

${ }^{3}$ Institute for Theoretical Physics, University of Amsterdam, 1090 GL Amsterdam, The Netherlands

${ }^{4}$ H. H. Wills Physics Laboratory, University of Bristol, Bristol BS8 1TL, UK

${ }^{5}$ Physical Sciences and Engineering, Argonne National Laboratory, Argonne, Illinois 60439, USA

${ }^{6}$ Division of Physics, Mathematics, and Astronomy, California Institute of Technology, Pasadena, California 91125, USA

Leading Summary: Charge ordering in metals is a fundamental instability of the electron sea, occurring in a host of materials and often linked to other collective ground states such as superconductivity. What is difficult to parse, however, is whether the charge order originates among the itinerant electrons or whether it arises from the ionic lattice. Here we employ high-resolution x-ray diffraction, combined with high pressure and low temperature techniques and theoretical modeling, to trace the evolution of the ordering wave vector $Q$ in charge and spin density wave systems at the approach to both thermal and quantum phase transitions. The non-monotonic behavior of $Q$ with pressure and the limiting sinusoidal form of the density wave point to the dominant role of the itinerant instability in the vicinity of the critical points, with little influence from the lattice. Fluctuations rather than disorder appear to disrupt coherence.

\section{Text:}

Understanding the formation and evolution of instabilities in electronic systems, from charge and spin order [1-6] to heavy fermion and high- $T_{c}$ superconductivity $[7,8]$, to insulator-metal transitions [9], has assumed a central stance in physics for decades. These different ordering mechanisms can compete and coexist, for instance charge and spin order vying with superconductivity in both normal [10] and high- $T_{\mathrm{c}}$ materials $[1,3$, 4], enriching the phase space of possible physical states. At the same time, isolating the fundamental mechanisms underlying a particular Fermi surface instability becomes key to progress in the field. At stake is not simply Fermiology, but the role of quantum critical points and the emergence of non-Fermi-liquid behavior [11, 12]. Perhaps the quintessential example, going back to the work of Peierls [13] in the 1950s, is the ongoing debate about the origin of charge-density-wave ordering in numerous compounds. Suggested mechanisms range from Fermi surface nesting and related instabilities in the electronic structure [14-16] to mediation by electron-phonon coupling effects $[17,18]$, to more exotic phenomena such as excitons paired through the screened Coulomb interaction $[19,20]$. 
In the case of charge (CDW) and spin (SDW) density waves, a continuous gap opening at the Fermi surface at zero temperature is complicated by the presence and potential influence of quantum critical fluctuations. Over the past two decades, there have been several theoretical approaches on quantum phase transitions with a $2 k_{\mathrm{F}}$ type of instability [16, 21-23]. This work has mainly focused on cuprates in two dimensions, and differ primarily on subtle issues such as distinguishing between nesting or hot spots, treating curved Fermi surfaces with parallel or non-parallel electron velocities, and modeling commensurate $(1 / 2,1 / 2)$ vs. incommensurate states. Given the presence of both bosonic and fermionic degrees of freedom in the theoretical framework, it is still unclear how the $2 k_{\mathrm{F}}$ type quantum phase transition becomes first [16] or second [23] order.

Experimental insight into the evolution of the electronic gap at the Fermi surface is typically provided by angle-resolved photoemission spectroscopy (ARPES) [24], measurement of the Hall coefficient $[11,25]$, or the observation of quantum oscillations [8]. It is important to note, however, that the wave vectors of the incommensurate CDW/SDW order also directly reflect the location and evolution of the associated energy gap in reciprocal space. With improved x-ray diffraction techniques applied over an extended pressure range $[6,26]$, it is now possible to finely resolve the evolution of incommensurate wave vectors and the role of an itinerant instability at a pressure-driven quantum critical point. This is a topic rarely discussed in the literature mainly because direct measurement of the evolution of the wave vector $Q$ by either neutron or x-ray diffraction was previously scarce, limited to low pressures, and of low resolution [27, 28]. Here, using diffraction-resolved incommensurate orders of the $\mathrm{CDW}$ in $\mathrm{NbSe}_{2}$ and the SDW in $\mathrm{Cr}$, we examine the fundamental physics of density wave formation approaching a pressure-driven critical point. We find that an itinerant electron instability plays a dominant role when the order parameter forms, taking on a pure sinusoidal shape unaffected by interactions with other degrees of freedom, and dictates the incipient longrange order at both the thermal and quantum critical points.

\section{Incommensurate wave vector in $P$ - $T$ space}

We plot in Fig. 1 both the Pressure-Temperature $(P-T)$ phase diagram and $Q(T, P)$ for our two model systems: the $\mathrm{CDW}$ in $2 \mathrm{H}-\mathrm{NbSe}_{2}$ and the $\mathrm{SDW}$ in $\mathrm{Cr}$. Although of different dimensions (two and three dimensions, respectively), the phase behavior of these two density-wave systems is similar (Fig. 1 insets). Pressure monotonically suppresses $T_{\mathrm{DW}}$, and both quantum phase transitions under pressure have proved to be continuous, with signatures of critical behavior in both electrical transport and x-ray diffraction $[2,5,25]$.

The evolution of $Q(P)$ over a $1.5 \%$ reduction in the lattice constant $[2,5]$ is nonmonotonic in the zero temperature limit, in contrast to the monotonic trend in $T_{\mathrm{DW}}(P)$. As we demonstrate below, the ordering wave vector $Q$ at the critical point represents an instability in the electronic structure, corresponds to a maximum in the full electronic susceptibility [21], and may be related to special features of the electronic bands or its Fermi surface [14-16]. In real systems with a spatial dimension higher than one, an itinerant instability is typically induced by a bosonic coupling between electronic states at 
the Fermi surface [21], with types of exchanged boson ranging from photon (Coulomb) $[14,19]$, phonon [17-18], to magnon (spin fluctuations) [6, 29]. The required finite strength of bosonic coupling has a limiting behavior towards zero only for perfect nesting, which is exemplified by the original Peierls' scenario of $2 k_{\mathrm{F}}$ instability of free electron gas in one dimension [13]. With the long-range DW order arising, the value of $Q$ may be tuned by physical processes beyond the band structure, resulting in nonmonotonic behavior in the $P-T$ phase space.

\section{Mean-field evolution of $Q(T, P=0)$}

Before discussing the $Q(P)$ behavior in detail, we survey $Q(T)$ of several $\mathrm{CDW} / \mathrm{SDW}$ materials at ambient pressure. The materials listed in Fig. 2a have incommensurate ordering existing to the lowest temperature and represent a diverse spectrum of spin or charge origins in various dimensions from one $\left(\mathrm{NbSe}_{3}\right)$ to three $(\mathrm{Cr}$, GdSi). Some, such as $\mathrm{TbTe}_{3}[30], \mathrm{Cr}$, and GdSi [6, 29], have well established nesting conditions at the Fermi surface, while others such as $\mathrm{NbSe}_{2}$ are at best described as hot spots [24]. Nevertheless, all five SDW/CDW states are clearly tied to instabilities of the itinerant electrons, resulting in the formation of gaps in the Fermi surface, with both resistivity anomalies [25, 29-32] and strong changes in the Hall coefficient $[29,33]$ at the transition. By contrast to $Q(P)$, all of the $Q(T)$ of the CDW/SDW systems in Fig. 2a evolve monotonically with temperature.

McMillan prescribed key elements of a mean-field free-energy expression for an incommensurate CDW/SDW [34]. Here we take the essence of the McMillan theory and neglect both interlayer and multi-Q interactions between CDWs. They are not a common feature of the examples listed above and thus are not expected to be necessary for explaining the $Q(T)$ behavior. The free energy density is then [2, 34]:

$$
F=a \psi^{2}+b \psi^{4}+c \xi^{2}|\nabla \psi|^{2}+e \psi^{2}|\nabla \phi-q|^{2}-f \psi^{n} \cos (n \phi-G x)
$$

The CDW order parameter $\operatorname{Re}\left(\psi e^{-i \phi}\right)$ has amplitude $\psi$ and phase $\phi . q$ is the natural wave vector of the $\mathrm{CDW}$ determined by a maximum in its full electronic susceptibility, and $\xi$ is the spatial correlation length of the CDW. $G$ is a reciprocal lattice unit vector. The first two terms are leading orders in a Landau free-energy expansion. The third and fourth terms come from the energy costs of distorting a CDW from its ideal condition by stretching/compressing the wave from the wave vector set by the susceptibility, and from having a finite spatial correlation length $\xi$. For CDWs, these two terms includes both electronic energy from repopulating the Brillouin zone [34] and static phonon energy from coupling to the lattice. The fifth term reflects the lock-in effect, which favors a CDW commensurate with the lattice. $Q(T)$ is determined by the competition between the fourth and fifth terms, as $\psi$ grows with decreasing temperature.

This competition is best visualized by plotting $Q(\psi)$ (Fig. 2b), using as examples TTF-TCNQ and $2 \mathrm{H}-\mathrm{TaSe}_{2}[35,36]$. Both CDW systems order incommensurately at high temperature, before experiencing a first-order phase transition to a commensurate state with discontinuities in both $Q$ and $\psi$. As shown in Fig. 2b, $Q$ is independent of $\psi$ in the $\psi$ 
$=0$ limit. As $\psi$ increases, $Q$ evolves continuously until the lock-in transition creates discontinuities in both $Q$ and $\psi$. At even lower temperature, $\psi$ continues to grow while $Q$ remains constant at the commensurate position. For TTF-TCNQ and $\mathrm{TaSe}_{2}, \mathrm{dQ} / \mathrm{d} \psi$ approaches zero at both small and large $\psi$, which clearly indicates the alternating dominance of either the fourth or fifth term in Eq. 1 with different powers of $\psi$. As exemplified by TTF-TCNQ, the lock-in position can be located far from the intrinsic CDW wave vector $q=Q\left(T_{\mathrm{DW}}\right)$, and $\Delta Q$ can be as large as one quarter of the reciprocal lattice unit [35]. In addition, a lock-in transition does not necessarily exist for every $n$ value; for TTF-TCNQ, the $Q(T)$ evolution passes through $n=3$ and locks in for $n=4$. This possibly explains the wave vectors in $\mathrm{NbSe}_{3}, \mathrm{GdSi}$, and $\mathrm{Cr}$ seemingly moving away from commensurate positions of $n=4,2$, and 1, respectively. For $\mathrm{NbSe}_{2}, n=3$ was assumed to be the commensurate position, in association with the lock-in transition in $\mathrm{TaSe}_{2}$ [34].

For CDWs that remain incommensurate, the $Q(\psi)$ plot in Fig. 2c only manifests partial features of the full evolution demonstrated in Fig. 2b. This indicates that the magnitude of $\psi$ is insufficient for higher order terms to contribute substantially to the free energy. $Q(\psi)$ behavior similar to Fig. 2c is also observed for the SDW in $\mathrm{Cr}$ at ambient $P$ (Fig. 3a of Ref. [2]). Hence $Q(\psi)$ provides a direct measure of the relative importance of terms with different powers of $\psi$ for a collection of vastly different density-wave systems. We note that the SDW in Cr possesses no local spin moments, and that SDW states in $\mathrm{Cr}$ and GdSi are not coupled to a lattice distortion at the primary wave vector $Q$ $[29,37]$. Thus the similar limiting behavior of $Q(\psi)$ at $\psi=0$, regardless of SDW or CDW type and coupling mechanism [6, 14, 17-19, 29] as shown in Fig. 2 and Ref. [2], points to an origin of these instabilities among the itinerant electrons rather than the localized ionic lattice. In general, $Q(\psi)$ provides a macroscopic indicator independent of the microscopic and quantitative details of the DW-lattice coupling. This is reflective of the fact that all $Q(T)$ in Fig. 2 behave similarly despite a variation of over a decade in the ordering temperature $T_{\mathrm{DW}}$.

\section{Harmonics of the incommensurate density wave}

For a sinusoidal incommensurate DW with both $\psi(x)=$ constant and $\phi(x)=Q x$, the last term in Eq. $1, f \psi^{n} \cos (n \phi-G x)$, always will be zero when summed over all lattice sites, no matter the magnitude of $\psi$. For a CDW and a collinear SDW, $\psi$ is a scalar and constant in a mean field framework [38], while all deviations of the DW from a pure sinusoid are represented by $\Delta \phi(x)=\phi(x)-Q x$. Experimentally, the deviation of an incommensurate wave from a sinusoidal form is reflected by the presence of higher order harmonics in the diffraction pattern $[37,39]$. For the CDW in $\mathrm{NbSe}_{2}$, we were able to measure both the primary wave and its second harmonic with our bulk-sensitive, highresolution x-ray diffraction technique (Fig. 3). Combining measurements at orders such as $(Q, 0,0),(1 \pm Q, 0,0)$, and $(2-Q, 0,0)$, the amplitude ratio between primary and second harmonics is $\psi_{2} / \psi_{1}=0.09 \pm 0.02$ at $T=3.5 \mathrm{~K}$. No other higher harmonic was observed within our measurement sensitivity (Fig. 3). Similar behavior in higher harmonics was observed in the SDW of $\mathrm{Cr}$ up to $4^{\text {th }}$ order of the primary wave [37]. For $\mathrm{Cr}$ in the low temperature limit, the amplitude ratios for SDW/CDW and their higher harmonics are 
$\psi_{3} / \psi_{1}=0.018$ and $\psi_{4} / \psi_{2}=0.019 \pm 0.003$, respectively [37], only a factor of five weaker than the ratio $\psi_{2} / \psi_{1}$ in $\mathrm{NbSe}_{2}$.

The deviation from pure sinusoidal behavior, $\Delta \phi(x)$, is chosen to minimize the free energy in Eq. 1. One possible solution was proposed by McMillan [40] as a "discommensuration" state, which has been widely considered as a model for the CDW in $\mathrm{NbSe}_{2}$. Locally, the CDW state is forced into a perfectly commensurate wavelength of $3 a$ by its coupling to the atomic lattice. In order to satisfy the requirement of having an incommensurate wave on average, the density modulations then develop phase slips of $2 \pi / 3$ between neighboring unit cells at every distance $\xi_{0}[40]$. To create the specific $Q$ $=1 / 3-\delta=0.3286$ r.l.u. observed at $T=3.5 \mathrm{~K}$ for $\mathrm{NbSe}_{2}$, the $-2 \pi / 3$ phase slips need to be placed at every $1 /(3 \delta) \sim 70$ unit cells, or a distance $\xi_{0} \sim 240 \AA$. To have an incommensurate order with a longer wavelength than that of the commensurate order $1 / 3$ r.l.u., all phase slips also need to have the same value of $-2 \pi / 3$ and no phase slips of $2 \pi / 3$ are allowed. A perfectly sharp $-2 \pi / 3$ phase slip creates many strong higher harmonics that would be readily observable, with amplitude ratios such as $\psi_{2} / \psi_{1}=0.45$ for first and second harmonics (Fig. 3b). To get agreement with the observed intensity ratios in $\mathrm{NbSe}_{2}$, we thus find that each phase slip is spread over a distance of the same order of magnitude as $\xi_{0}$. Our measurements show that the modulation of the electronic density in the bulk is closer to that of a perfectly incommensurate pattern than the discommensuration form with sharp phase slips proposed by McMillan and observed in the surface state of $\mathrm{NbSe}_{2}$ [41].

Approaching the thermal transition temperature, $T_{\mathrm{DW}}$, the diminishing coupling between the CDW and the lattice is reflected directly in the harmonic behavior. In Fig. 4, we plot representative diffraction profiles and the temperature evolution of the CDW at $(1+Q, 0,0)$. The intensities of both the primary and secondary harmonics of the CDW in $\mathrm{NbSe}_{2}$ decrease with increasing $T$. However, the intensity of the second harmonic drops much faster than that of the primary CDW, with a relative ratio between the two evolving in a nearly linear fashion in temperature (Fig. 4 inset). Similar behavior was seen as well in $\mathrm{Cr}$, where the higher harmonic intensities scale as $I_{\mathrm{Q}}(T) \sim I_{2 \mathrm{Q}}{ }^{1 / 2} \sim I_{4 \mathrm{Q}}{ }^{1 / 4}$ [37]. We conclude that the density waves due to the itinerant instabilities in $\mathrm{NbSe}_{2}$ and $\mathrm{Cr}$ both have the limiting form of a purely sinusoidal wave at $T_{\mathrm{DW}}$, with a $\Delta \phi(x) \rightarrow 0$.

\section{Evolution of a non-monotonic $Q(T=0, P)$}

The non-monotonic behavior of $Q(T=0, P)$ in Fig. 1 evolves over a range of 0.003-0.012 r.l.u., which is well below the resolution of photoemission techniques [24]. As density functional theory approaches are still insufficient to describe the SDW ground state in $\mathrm{Cr}$ [42], this subtle non-monotonic behavior is difficult to resolve with $a b$ initio calculations. Here instead, we argue that $Q(P)$ can be explained by macroscopic free energy considerations similar to those made for $Q(T)$ at ambient $P$, once the pressure dependence of the different contributions to the electronic susceptibility are taken into account. 
As discussed above, the temperature evolution of the incommensurate wave vector $Q$ starts at the itinerant condition $q$ at $T_{\mathrm{DW}}$ and changes to $Q(T=0, P)$ due to the higher-order lock-in term that pulls $Q$ away from $q$. The pressure dependence of $q$ also has been measured, as a part of the full $Q(T)$ evolution, at several low pressures up to 0.2 and $0.6 \mathrm{GPa}$ for $\mathrm{NbSe}_{2}$ and $\mathrm{Cr}$, respectively $[27,28]$. Those studies provide valuable information of initial changes in $q(P)=Q\left(T_{\mathrm{DW}}, P\right)$. From Fig. $1, q(P)$ is clearly nonlinear, but nevertheless is still likely monotonic, given the small variation of $1.5 \%$ in the lattice constant. When the transition temperature $T_{\mathrm{DW}}$ decreases towards the quantum critical point, the strength of the order parameter $\psi$ monotonically decreases with $P$ at zero temperature. The competition between the lower-order electronic instability term and the higher-order lock-in term in Eq. 1 becomes increasingly one-sided with reducing $\psi$ under pressure. This is consistent with the experimental observation that $\Delta Q(P)=q(P)$ $Q(T=0, P)$ decreases in magnitude under pressure in both $\mathrm{NbSe}_{2}$ and $\mathrm{Cr}[27,28]$ (Fig. 1). Eventually, close to the quantum critical point the lower-order term $\psi^{2}|\nabla \phi-q|^{2}$ in Eq. 1 dominates, and $q(P)$ and $Q(T=0, P)$ converge at $P_{c}$. The non-monotonic behavior in $Q(T=0, P)$ is therefore due to a changing balance between terms of various powers in $\psi$, in addition to a varying $q$ under pressure. The measured CDW/SDW wave vector at the quantum critical point is a true reflection of a maximum in the full electronic susceptibility arising from features such as (but not limited to) the presence of nesting or hot spots in the electronic structure.

We model in Fig. 5 the $Q(T, P)$ behavior for $\mathrm{NbSe}_{2}$ using the free energy expression in Eq. 1, with parameters and coefficients constrained by physical considerations in the following way. For $a(T, P)$, which determines $T_{\mathrm{CDW}}$, its temperature dependence is assumed to be linear, while its pressure dependence is determined from the measured phase diagram: $a(T, P)=-a_{0}\left(1-T / T_{\mathrm{CDW}}(P)\right)$. The coefficient $b(T, P)$ for the quartic term is assumed constant, which determines the primary CDW amplitude at $T=0$ through the ratio $b / a$. Although the spatial correlation length $\xi$ is pressure dependent [5], the third term in Eq. 1 describing spatial variations of the DW amplitude is neglected in our modeling since it does not directly affect $Q$. The pressure dependence of the fourth term is directly taken from experimental data as $q(P)=Q\left(T_{\mathrm{DW}}, P\right)$ with coefficient $e$ assumed constant. The pressure dependence of the coefficient $f$ of the lock-in term, which arises from the variation of orbital overlaps with pressure, is assumed linear given the small change in the compressed lattice constant. We use McMillan's discommensuration construction to build the phase distortion $\Delta \phi(x)$ (Supplementary Information). The width and density of phase slips in $\phi(x)$ can be varied to minimize the free energy $F$ of Eq. 1 and the density of phase slips generates the $Q$ value at every point in $P-T$ phase space.

Apart from an overall scaling factor for the magnitude of the free energy, there are only two free parameters left. They describe the ratio of the lock-in energy to the energy cost of deviating from $q$ set by the fourth term in Eq. 1, as well as the slope of the linear pressure dependence in the lock-in term. While $\Delta \phi(x)$ minimizes the free energy and determines $Q$ at every $P-T$ point, optimizing those two parameters results in the global behavior of $Q(T, P)$ in Fig. 5 (Supplementary Information). The qualitative agreement with experimental results in both the magnitude of $Q$ variations, as well as the 
non-monotonic pressure dependence, is satisfying given the simplicity of approximations used.

\section{$2 k_{F}$ instability at a quantum critical point}

In our current study, the CDW state in $\mathrm{NbSe}_{2}$ was tracked under pressure (Fig. 1, Fig. 6) up to the quantum phase boundary at $P_{\mathrm{c}}=4.6 \mathrm{GPa}$ [5]. In addition to wave vector $Q(T, P)$, x-ray diffraction also provides the static CDW correlation length $\xi$ in real space, with $\xi=600 \AA$ for our samples at $T=3.5 \mathrm{~K}$ and ambient pressure (Fig. 6). While the lattice correlation length remains long range to at least $1500 \AA$ throughout our probed $P-T$ space, $\xi$ gradually decreases as it approaches both the thermal and quantum critical points [5], similar to thermal behavior in other SDW/CDWs [37, 43]. Near both thermal and quantum phase boundaries, $\xi$ is about 26-50 $\AA$ in $\mathrm{NbSe}_{2}$ (Fig. 6), roughly 3-5 CDW wave lengths or 8-15 unit cell sizes.

The mechanism for destroying long-range static CDW coherence, leading to a shortened $\xi$, has been attributed to an increase in either fluctuation effects [5] or disorder pinning $[37,38,43]$. At $T=3.5 \mathrm{~K}$ and $P=0$, our observed CDW line shape is described by a Lorentzian-squared function (Figs. 3b, 6c), which is consistent with disorder pinning the CDW to random phases at impurity sites [5]. A strong pinning picture is unlikely to apply near the transition, since it necessarily introduces many higher harmonics of the CDW, by contrast to our observed temperature evolution (Fig. 4). Approaching either the thermal or quantum phase transitions, the line shape is best described by a pure Lorentzian form (Fig. 6a) [5, 43], which indicates that the CDW phase correlation is exponentially decaying in real space without experiencing abrupt changes. While this could be consistent with a pinning picture that allows the CDW phase distortion to be distributed over a spatial range across a pinning site [38], the extremely short $\xi$ of 8-15 unit cells in our observation is unlikely to host multiple disorder sites within a coherent volume, necessary to collectively anchor the phase-coherent CDW domain. We conclude that the shortened correlation lengths are more likely due to increasing (quantum or thermal) fluctuation effects at the phase boundaries. Furthermore, $\phi(x)$ is not constrained by disorder, but has the freedom to adjust to the itinerant instability and the observed incommensurate CDW wave vector $\boldsymbol{Q}$ continuously evolves approaching both the thermal and quantum limits (Fig. 1). Similarly, CDWs remain incommensurate with very short correlation lengths in cuprates [3, 4], despite significant disorder from doping. Our observation of a sinusoidal, incommensurate density wave and a short correlation length signifies respectively the importance of itinerant instability and fluctuation effects at both classical and quantum critical points.

Our study examines $2 k_{\mathrm{F}}$ density waves in solid-state materials from a top-down perspective. Pressure tuning helps to identify the itinerant instability as the dominant influence at a quantum critical point, even in the presence of spin, charge, orbital, and lattice degrees of freedom. The striking similarity between SDW and CDW systems in both $Q(\psi)$ at ambient $P$ (Fig. 2 and Ref. [2]) and $Q(T, P)$ over $P-T$ space (Fig.1) clearly manifests the close relationship between these two types of correlated electron states, regardless of the presence of instabilities among the local ions or spins, or the lack 
thereof. Our work should motivate attempts to reconcile theories that describe charge and spin instabilities from very different points of view. CDWs originating from itinerant electrons previously have been pooled indifferently with incommensurate lattice systems [18]. However, the wave vectors of incommensurate lattice distortions tend to be temperature independent [44]. Based on the observed evolution of $Q(T, P)$ and $Q(\psi)$, our comparative study of CDWs and SDWs emphasizes the universal nature and importance of correlated itinerant pairs, rather than lattice instabilities, in the presence of various choices of coupling mechanisms. In CDWs, even if the itinerant instability is mediated by the Coulomb interaction (photons), there should still exist additional phonon coupling because of the presence of the localized charge lattice [18]. For spins, phonons become largely irrelevant in SDWs both with and without local moments [29, 37], mirroring the charge scenario. Directly resolving boson coupling between itinerant electron pairs in traditional CDW/SDW systems or high temperature cuprates remains challenging [45], and time-resolved techniques such as pump-probe ARPES [46] potentially could provide key insights into the different contributors to the full electronic susceptibility.

In complementary experiments, Fermi surfaces with a nesting instability potentially could be manufactured from a bottom-up approach using cold atoms. Following the early idea of a commensurate nesting condition [47], recent developments in shaken optical lattices [48] have pointed to a method to produce customized band structures with incommensurate nesting. Since phonons are not naturally present in an optical lattice, the coupling between paired atoms instead comes from the short-range Van der Waals interaction, which is nearly $q$-independent in reciprocal space and could be tuned to be attractive. This is comparable to various interactions between itinerant pairs in solids that are not phonon-mediated, such as the screened Coulomb interaction in excitons [19, 20] or magnons from fluctuations in locally ordered spins [29]. Alternatively, the electron-phonon interaction may be mirrored in a mixture of Fermionic and Bosonic atoms in a weak optical potential. A comparison of these different constructions in cold atom systems could shed additional light on itinerant instabilities at quantum critical points $[14,17,18]$.

\section{Methods}

$\mathrm{X}$-ray diffraction. Both ambient and high-pressure $\mathrm{x}$-ray diffractions were performed at beamline 4-ID-D of the Advanced Photon Source. $18.85 \mathrm{keV}$ x-rays were used, chosen to lie below the $\mathrm{Nb} K$-edge to avoid this fluorescence excitation. The x-ray beam was focused to a FWHM size of $250 \times 120(\mathrm{H} \times \mathrm{V}) \mu \mathrm{m}^{2}$ using Pd coated mirrors and further narrowed by motorized slits for high-pressure work. For high-resolution longitudinal scans, a vertical detector slit size of 0.1 or $0.2 \mathrm{~mm}$ was used, with a sample to slit distance of $1.25 \mathrm{~m}$. Diffraction in the vertical plane thus had a maximal momentum-space resolution of about $110^{-3} \AA^{-1}$. Both ambient and high-pressure diffraction were performed in the transmission geometry to the $\boldsymbol{a}-\boldsymbol{b}$ plane of $\mathrm{NbSe}_{2}$ for bulk sensitivity. At ambient pressure, a Vortex ${ }^{\circledR}$ Si-drift detector was used to reject Se $K$-fluorescence. For the high-pressure study, a Cyberstar NaI x-ray detector was used for a higher counting efficiency, as the diamond anvils absorb the majority of the Se $K$-fluorescence. Both $\mathrm{x}$ ray detectors only have coarse energy resolutions of $0.1-1 \mathrm{keV}$. However, the spanned

solid angle of 1-3 $10^{-7} \mathrm{sr}$ by our detector slits effectively eliminated the detected intensity 
of inelastic scattering of dynamic CDW fluctuations. Thus our observed CDW diffraction near the quantum critical point represents the static order, in comparison to the quasielastic nature of resonant $\mathrm{x}$-ray scattering techniques [3].

Sample and high-pressure environment. Single crystals of $\mathrm{NbSe}_{2}$ were used in asgrown condition at ambient pressure. The thickness of ambient pressure samples, varying from 40 to $75 \mu \mathrm{m}$, matches well with one x-ray absorption length of our x-rays. For high-pressure measurements, single crystals were prepared by blade dicing to a typical size of $80 \times 80 \times 50 \mu^{3}$. A methanol:ethanol $4: 1$ mixture was used as the pressure medium. More details can be found in Refs. [5, 26].

\section{References:}

[1] Tranquada, J.M., Sternlieb, B.J., Axe, J.D., Nakamura, Y. \& Uchida S., Evidence of stripe correlations of spins and holes in copper oxide superconductors. Nature 375, 561563 (1995).

[2] Jaramillo, R. et al. Breakdown of the Bardeen-Cooper-Schrieffer ground state at a quantum phase transition. Nature 459, 405-409 (2009).

[3] Ghiringhelli, G. et al., Long-range incommensurate charge fluctuations in (Y,Nd)Ba $\mathrm{Cu}_{3} \mathrm{O}_{6+\chi}$. Science 337, 821-825 (2012).

[4] Chang, J. et al., Direct observation of competition between superconductivity and charge density wave order in $\mathrm{YBa}_{2} \mathrm{Cu}_{3} \mathrm{O}_{6.67}$. Nat. Phys. 8, 871-876 (2012).

[5] Feng, Y. et al., Order parameter fluctuations at a buried quantum critical point. Proc. Nat. Acad. Sci. USA 109, 7224-7229 (2012).

[6] Feng, Y. et al., Hidden one-dimensional spin modulation in a three-dimensional metal, Nat. Communs. 5:4218 (2014).

[7] Pfleiderer, C. Superconducting phases of f-electron compounds. Rev. Mod. Phys. 81, 1551-1624 (2009).

[8] Doiron-Leyraud et al., Quantum oscillations and the Fermi surface in an underdoped high- $T_{\mathrm{c}}$ superconductor. Nature 447, 565-568 (2007).

[9] Imada, M., Fujimori, A. \& Tokura, Y. Metal-insulator transitions. Rev. Mod. Phys. 70, 1039-1263 (1998).

[10] Littlewood, P.B. \& Varma, C.M. Gauge-invariant theory of the dynamical interaction of charge density waves and superconductivity. Phys. Rev. Lett. 47, 811-814 (1981).

[11] Paschen, S. et al., Hall-effect evolution across a heavy fermion quantum critical point. Nature 432, 881-885 (2004).

[12] Löhneysen, H. V., Rosch, A., Vojta, M. \& Wölfle, P. Fermi-liquid instabilities at magnetic quantum phase transitions. Rev. Mod. Phys. 79, 1015-1075 (2007).

[13] Peierls, R. E. Quantum Theory of Solids, Oxford Univ. Press, Oxford (1955).

[14] Overhauser, A.W. Spin density waves in an electron gas. Phys. Rev. 128, 1437-1452 (1962).

[15] Rice, T. M. \& Scott, G. K. New Mechanism for a Charge-Density-Wave Instability. Phys. Rev. Lett. 35, 120-123 (1975).

[16] Abanov, Ar. \& Chubukov, A. V. Spin-fermion model near the quantum critical point: one-loop renormalization group results. Phys. Rev. Lett. 84, 5608-5611 (2000). 
[17] Chan, S.-K. \& Heine, V. Spin density wave and soft phonon mode from nesting Fermi surfaces. J. Phys. F: Met. Phys. 3, 795-809 (1973).

[18] Johannes M.D. \& Mazin, I.I. Fermi surface nesting and the origin of charge density waves in metals. Phys. Rev. B 77, 165135 (2008).

[19] Jérome, D., Rice, T.M. \& Kohn, W. Excitonic insulator. Phys. Rev. B 158, 462-475 (1967).

[20] van Wezel, J., Nahai-Williamson, P. \& Saxena, S. S. Exciton-phonon-driven charge density wave in $\mathrm{TiSe}_{2}$. Phys. Rev. B 81, 165109 (2010).

[21] Altshuler, B. L., Ioffe, L. B. \& Millis, A. J. Critical behavior of the $T=02 k_{\mathrm{F}}$ density-wave phase transition in a two-dimensional Fermi liquid. Phys. Rev. B 52, 55635572 (1995).

[22] Metlitski, M. A. \& Sachdev, S. Quantum phase transitions of metals in two spatial dimensions. II. Spin density wave order. Phys. Rev. B 82, 075128 (2010).

[23] Bergeron, D., Chowdhury, D., Punk, M., Sachdev, S. \& Tremblay, A.-M. S. Breakdown of Fermi liquid behavior at the $(\pi, \pi)=2 k_{\mathrm{F}}$ spin-density wave quantumcritical point: The case of electron-doped cuprates. Phys. Rev. B. 86, 155123 (2012).

[24] Borisenko, S.V. et al. Two energy gaps and Fermi-surface "arcs" in $\mathrm{NbSe}_{2}$. Phys. Rev. Lett. 102, 166402 (2009).

[25] Jaramillo, R., Feng, Y., Wang, J. \& Rosenbaum, T. F. Signatures of quantum criticality in pure $\mathrm{Cr}$ at high pressure. Proc. Nat. Acad. Sci. USA 107, 13631-13635 (2010).

[26] Feng, Y., Jaramillo, R., Wang, J., Ren, Y. \& Rosenbaum, T. F. High-pressure techniques for condensed matter physics at low temperature. Rev. Sci. Instrum. 81, 041301 (2010).

[27] Umebayashi, H., Shirane, G., Frazer, B.C. \& Daniels, W.B. Neutron diffraction study of Cr under high pressure. J. Phys. Soc. Jap. 24, 368-372 (1968).

[28] McWhan, D. B., Fleming, R. M., Moncton, D. E. \& DiSalvo, F. J. Reentrant lock-in transition of the charge-density wave in $2 \mathrm{H}-\mathrm{TaSe}_{2}$ at high pressure. Phys. Rev. Lett. 45, 269-272 (1980).

[29] Feng, Y. et al., Incommensurate antiferromagnetism in a pure spin system via cooperative organization of local and itinerant moments. Proc. Nat. Acad. Sci. USA 110, 3287-3292 (2013).

[30] Ru, N. et al., Effect of chemical pressure on the charge density wave transition in rare-earth tritellurides $R \mathrm{Te}_{3}$, Phys. Rev. B 77, 035114 (2008).

[31] Berthier, C., Molinié, P. \& Jérome, D. Evidence for a connection between charge density wave s and the pressure enhancement of superconductivity in $2 \mathrm{H}-\mathrm{NbSe}_{2}$. Solid State. Comm. 18, 1393-1395 (1976).

[32] P. Monçeau, N. P. Ong, A. M. Portis, A. Meerschaut, and J. Rouxel, Electric field breakdown of charge-density-wave induced anomalies in $\mathrm{NbSe}_{3}$. Phys. Rev. Lett. 37, 602606 (1976).

[33] Lee, H.N.S., McKinzie, H., Tannhauser, D.S. \& Wold, A. The low-temperature transport properties of $\mathrm{NbSe}_{2}$. J. Appl. Phys. 40, 602-604 (1969).

[34] McMillan, W. L., Microscopic model of charge-density-wave in $2 \mathrm{H}-\mathrm{TaSe}_{2}$. Phys. Rev. B 15, 643-650 (1977).

[35] Kagoshima, S., Ishiguro, T. \& Anzai, H. X-ray scattering study of phonon anomalies and superstructures in TTF-TNQ. J. Phys. Soc. Jap. 41, 2061-2071 (1976). 
[36] Moncton, D. E., Axe, J. D. \& DiSalvo, F. J. Neutron scattering study of the chargedensity wave transition in $2 \mathrm{H}-\mathrm{TaSe}_{2}$ and $2 \mathrm{H}-\mathrm{NbSe}_{2}$. Phys. Rev. B 16, 801-819 (1977).

[37] Hill, J. P., Helgesen, G. \& Gibbs, D. X-ray-scattering study of charge- and spindensity-waves in chromium. Phys. Rev. B 51, 10336-10344 (1995).

[38] Fukuyama, H. \& Lee, P.A. Dynamics of the charge-density wave. I. Impurity pinning in a single chain. Phys. Rev. B 17, 535-541 (1978).

[39] Jensen, J. \& Mackintosh, A.R. Helifan: A new type of magnetic structure. Phys. Rev. Lett. 64, 2699-2702 (1990).

[40] McMillan, W. L., Theory of discommensurations and the commensurateincommensurate charge-density-wave phase transition. Phys. Rev. B 14, 1496-1502 (1976).

[41] Soumyanarayanan, A. et al., Quantum phase transition from triangular to stripe charge order in $\mathrm{NbSe}_{2}$. Proc. Nat. Acad. Sci. USA 110, 1623-1627 (2013).

[42] Vanhoof, V., Rots, M. \& Cottenier, S. Spin-density wave in Cr: Nesting versus lowlying thermal excitations. Phys. Rev. B 80, 184420 (2009).

[43] DiCarlo, D., Thorne, R.E., Sweetland, E., Sutton, M. \& \& Brock, J. D. Chargedensity-wave structure in $\mathrm{NbSe}_{3}$. Phys. Rev. B 50, 8288-8296 (1994).

[44] Schutte, W. J. \& De Boer, J. L. Valence fluctuations in the incommensurately modulated structure of Calaverite AuTe 2 . Acta Cryst. B44, 486-494 (1988).

[45] Sentef, M. et al., Examining electron-boson coupling using time-resolved spectroscopy. Phys. Rev. X 3, 041033 (2013).

[46] Schmitt, F. et al., Transient electronic structure and melting of a charge density wave in $\mathrm{TbTe}_{3}$. Science 321, 1649-1652 (2008).

[47] Makogon, D., Spielman, I. B. \& Smith, C. M., Spin-charge-density wave in a rounded-square Fermi surface for ultracold atoms. Euro. Phys. Lett. 97, 33002 (2012).

[48] Parker, C.V., Ha, L.-C. \& Chin, C. Direct observation of effective ferromagnetic domains of cold atoms in a shaken optical lattice. Nat. Phys. 9, 769-774 (2013).

[49] Feng, Y. et al. Energy dispersive x-ray diffraction of charge density waves via chemical filtering. Rev. Sci. Instrum. 76, 063913 (2005).

[50] Fleming, R.M., Moncton, D.E. \& McWhan, D.B. X-ray scattering and electric field studies of the sliding mode conductor $\mathrm{NbSe}_{3}$. Phys. Rev. B 18, 5560-5563 (1978).

[51] Moudden, A.H., Axe, J.D., Monceau, P. \& Levy, F. $q_{1}$ Charge-density wave in $\mathrm{NbSe}_{3}$. Phys. Rev. Lett. 65, 223-226 (1990).

[52] Fleming, R.M., Schneemeyer, L.F. \& Moncton, D.E. Commensurat-incommensurate transition in the charge-density-wave state of $\mathrm{K}_{0.30} \mathrm{MoO}_{3}$. Phys. Rev. B 31, 899-903 (1985).

\section{Acknowledgments}

We are grateful for stimulating discussions with R. Jaramillo, C. V. Parker, and M. R. Norman, and for $\mathrm{NbSe}_{2}$ samples provided by Y. Liu and Z.-A. Xu. The work at the University of Chicago was supported by National Science Foundation Grant No. 1206519. The work at the Advanced Photon Source of Argonne National Laboratory was supported by the U.S. Department of Energy Basic Energy Sciences under Contract No. NEAC02-06CH11357. J.v.W. acknowledges support from a VIDI grant financed by the Netherlands Organization for Scientific Research (NWO). 


\section{Author contributions}

Y.F. and T.F.R. conceived the research. Y.F. and J.W. performed X-ray measurements. Y.F., D.M.S., and T.F.R. analyzed the data. Y.F., J.v.W., F.F., and P.B.L. developed the theoretical framework. Y.F., J.v.W., and T.F.R. prepared the manuscript. All authors commented.

\section{Additional information}

Correspondence and requests for materials should be addressed to Y.F. (yejun@anl.gov) or T.F.R. (tfr@caltech.edu).

\section{Competing financial interests}

The authors declare no competing financial interests.

\section{Figure captions}

Figure 1 | Pressure-temperature evolution of incommensurate wave vector $Q$. For (a) $\mathrm{CDW}$ in $\mathrm{NbSe}_{2}$ and (b) SDW in Cr, the ordering wave vector $Q$ is plotted as a function of both temperature $T$ and pressure $P$. For $\mathrm{NbSe}_{2}, Q(T, P)$ with solid symbols are from the current study, while open symbols are from Refs. [28] and [36] with a shift of all $Q$ by 0.00095 r.l.u. to match our data at $Q(0,0)$. For $\mathrm{Cr}, Q(T, P)$ are collected from the literature (open symbols) [27] and our previous work (solid symbols) [2, 45]. (Insets) $P$ $T$ phase diagrams of $\mathrm{NbSe}_{2}[5,31]$ and $\mathrm{Cr}[25]$ show similar monotonic trends in $T_{\mathrm{DW}}(P)$. On the other hand, $Q(P)$ of $\mathrm{NbSe}_{2}$ and $\mathrm{Cr}$ manifest a non-monotonic behavior in the low temperature limit. Arrows in phase diagrams mark our measurement trajectories for data plotted in main panels.

Figure 2 | Temperature evolution of wave vector $\boldsymbol{Q}$. (a) Incommensurate $Q(T)$ for CDWs and SDWs arranged by increasing $T_{\mathrm{DW}}$ over one decade. (b) $Q(\psi)$, with temperature as the hidden variable in the range $0<T<T_{\mathrm{DW}}$, are plotted for the CDW systems TTF-TCNQ and $\mathrm{TaSe}_{2}$, both of which manifest a lock-in transition to a commensurate state. Here, $\psi$ at zero temperature is strong enough that $Q(\psi)$ shows the full evolution between the two limits of $\psi_{T} \psi_{T=0}=0$ and 1; each end is dominated by individual terms of different powers of $\psi$ in the Hamiltonian of Eq. 1. (c) $Q(\psi)$ for CDWs which remain incommensurate at low temperature only evolves over part of the master curve in Fig. 3b. $Q(T)$ of $\mathrm{NbSe}_{2}$ from the current study is compared to data from the literature: $\mathrm{NbSe}_{2}$ [36], GdSi [6, 29], $\mathrm{NbSe}_{3}$ [50, 51], $\mathrm{Cr}$ [49], $\mathrm{TbTe}_{3}$ [30], $\mathrm{TaSe}_{2}$ [36], TTF-TCNQ [35], and $\mathrm{K}_{0.3} \mathrm{MoO}_{3}$ [51]. The CDW amplitude $\psi(T)$ is obtained from x-ray or neutron diffraction intensity $I(T)$ by $I \sim \psi^{2}$ [37], and is normalized by its low temperature limit $\psi_{T=0}$ for comparisons in Figs. $2 \mathrm{~b}$ and $2 \mathrm{c}$. For $\mathrm{K}_{0.3} \mathrm{MoO}_{3}$, the experimental resolution was insufficient to determine whether or not a lock-in transition occurs; No first order discontinuity was observed in the temperature dependence of both the order parameter and the wave vector [52].

Figure 3 | CDW harmonics in NbSe $_{2}$. (a) Longitudinal $(\theta / 2 \theta)$ scan in reciprocal lattice unit (r.l.u.) along the in-plane direction of $(H, 0,0)$ for $\mathrm{NbSe}_{2}$. Both the primary and second harmonics of CDW are clearly resolved over the background around mirroring $(Q, 0,0)$ and $(1-Q, 0,0)$ positions. The plotted intensity is normalized to that of the $(1,0$, 
0) lattice order. Vertical error bars represent $1 \sigma$ s.d. of counting statistics. (b) A profile comparison between the experimental observed $\mathrm{CDW}$ for $\mathrm{NbSe}_{2}$ at $(Q, 0,0)$ (red points) and a simulated diffraction pattern of a discommensurate CDW with phase slips in the sharp limit (grey line) [40]. For comparison, both intensities are normalized to unity at the primary wave vector position. The fine longitudinal CDW scan in panel b was measured with our best instrument resolution (better by a factor of two than the long scan in Fig. 2a; Methods). The data is fit by the summation of two Lorentzian-squared functions for primary and second harmonics in addition to a constant background (red solid line). Positions of CDW harmonics are marked by black arrows on the horizontal axes in both panels a and $\mathbf{b}$, with the separation between the primary incommensurate CDW and the commensurate $(1 / 3,0,0)$ position (white circle) denoted as $\delta=1 / 3-Q$. The separation becomes $m \delta$ for the $m^{\text {th }}$ order harmonic; these are naturally translated by the unit vector of the lattice periodicity, and hence for small $\delta$ are observed close to the commensurate position. Harmonics from $4^{\text {th }}$ to $10^{\text {th }}$ orders are not observed even with a CDW signal-to-background ratio larger than 5000. The simulated diffraction profile for a discommensurate CDW with single-helicity $-2 \pi / 3$ phase slips over a single unit-cell at a regular spacing of 70 unit-cell distance has strong harmonic peaks at all orders within our measurement range [41].

Figure 4 | Temperature evolution of CDW harmonics in $\mathrm{NbSe}_{2}$. (Main) Representative longitudinal scans of $(1+Q, 0,0)$ and $(2-2 Q, 0,0)$ at four different temperatures below $T_{\mathrm{CDW}}$. (Inset) Temperature dependence of the intensity ratio between the second harmonic and the primary CDW, plotted for one single sample. The intensity ratio was fit (solid line) to a power law of $I_{(2-2 Q, 0,0)} / I_{(1+Q, 0,0)} \sim\left(T_{C D W}-T\right)^{2 \beta}$ with $\beta=0.5 \pm 0.1$, and $T_{\mathrm{CDW}}=36.8 \pm 3.3 \mathrm{~K}$.

Figure 5 | Theoretical modeling of $\boldsymbol{Q}(\mathbf{T}, \boldsymbol{P})$. The evolution of $Q(T, P)$ is modeled by minimizing the free energy in Eq. 1 for the $\mathrm{CDW}$ in $\mathrm{NbSe}_{2}$ with optimally spaced and widened phase slips, with the global behavior controlled by two free parameters. Calculated $Q(P)$ at several fixed temperatures (linked dots) are plotted in comparison with experimental data (grey crosses bound by grey dashed lines, from Fig. 1), capturing the non-monotonic behavior of $Q(P)$. The simulated results also show a lock-in transition at $T=0$ (horizontal dashed line).

Figure 6 | CDW fluctuations near the thermal and quantum critical points. Longitudinal x-ray diffraction scans are plotted for the $\mathrm{CDW}$ in $\mathrm{NbSe}_{2}$. At (a) $\left(T \sim T_{\mathrm{CDW}}\right.$, $P=0)$ and (b) $\left(T \rightarrow 0, P \sim P_{\mathrm{c}}\right)$, the CDW line shapes are best fit with a Lorentzian form plus a linear background (red, panel a; light blue, panel b). At (c) $(T \rightarrow 0, P=0)$, the fit to the CDW line shape instead requires the sum of two Lorentzian-squared functions and a constant background (blue, panel c). A Lorentzian-squared form with a linear background (black) is also used to fit data in panel a as a comparison. The vertical dashed lines mark the $1 / 3$ commensurate position. Locations of all three $(T, P)$ points are marked by asterisks of the same color on the grey $P-T$ phase diagram in the background. Near the phase boundary, the CDW profile represents a small CDW correlation length $\xi$, which is $\sim 50 \AA$ for $\left(T \sim T_{\mathrm{CDW}}, P=0\right)$ and $\sim 26 \AA$ for $\left(T \rightarrow 0, P \sim P_{\mathrm{c}}\right)$ in the basal plane. The shortened 
$\xi$ is inconsistent with pinning from disorder and is instead attributed to thermal fluctuations and quantum fluctuations [5] near their respective critical points. 

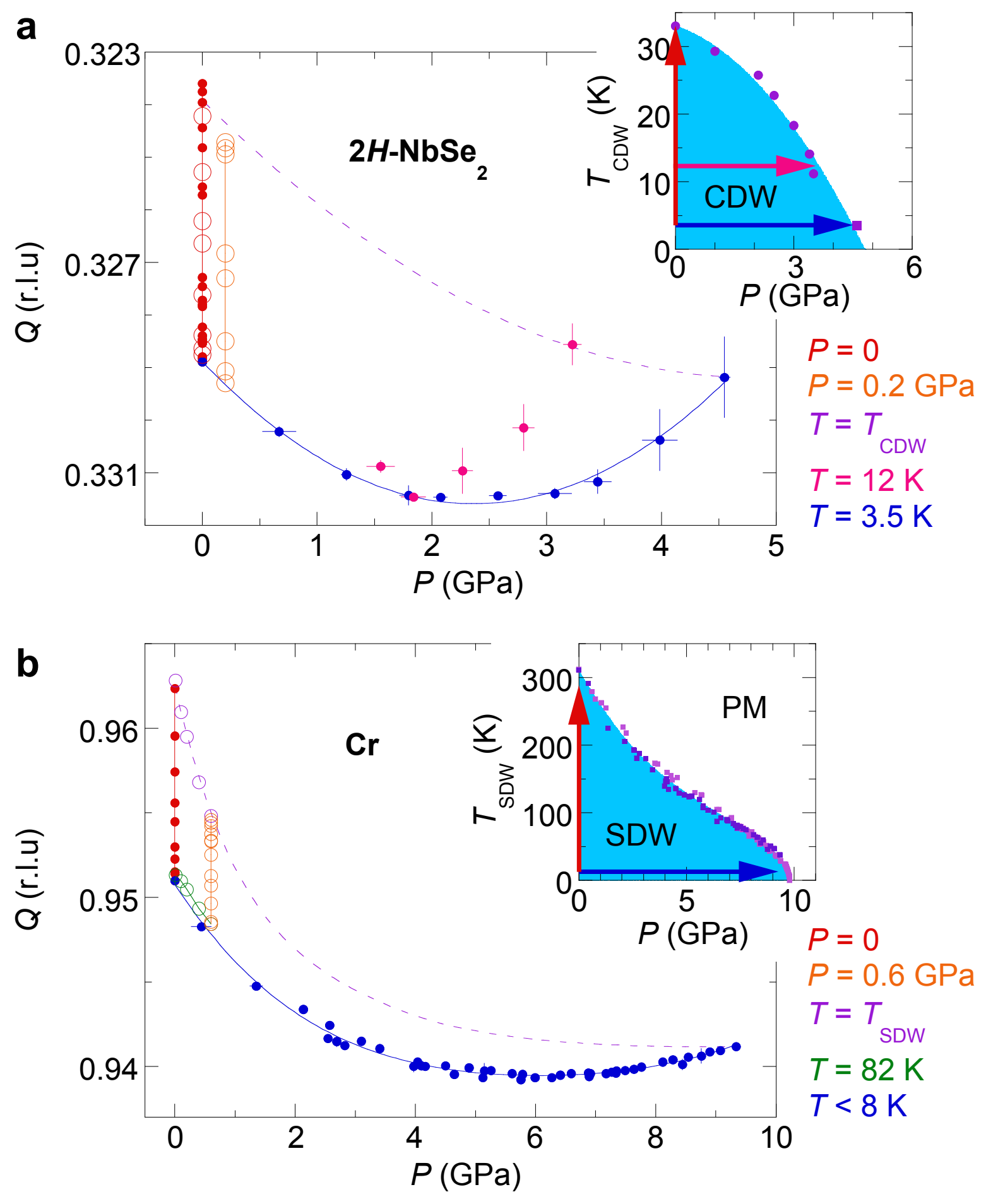

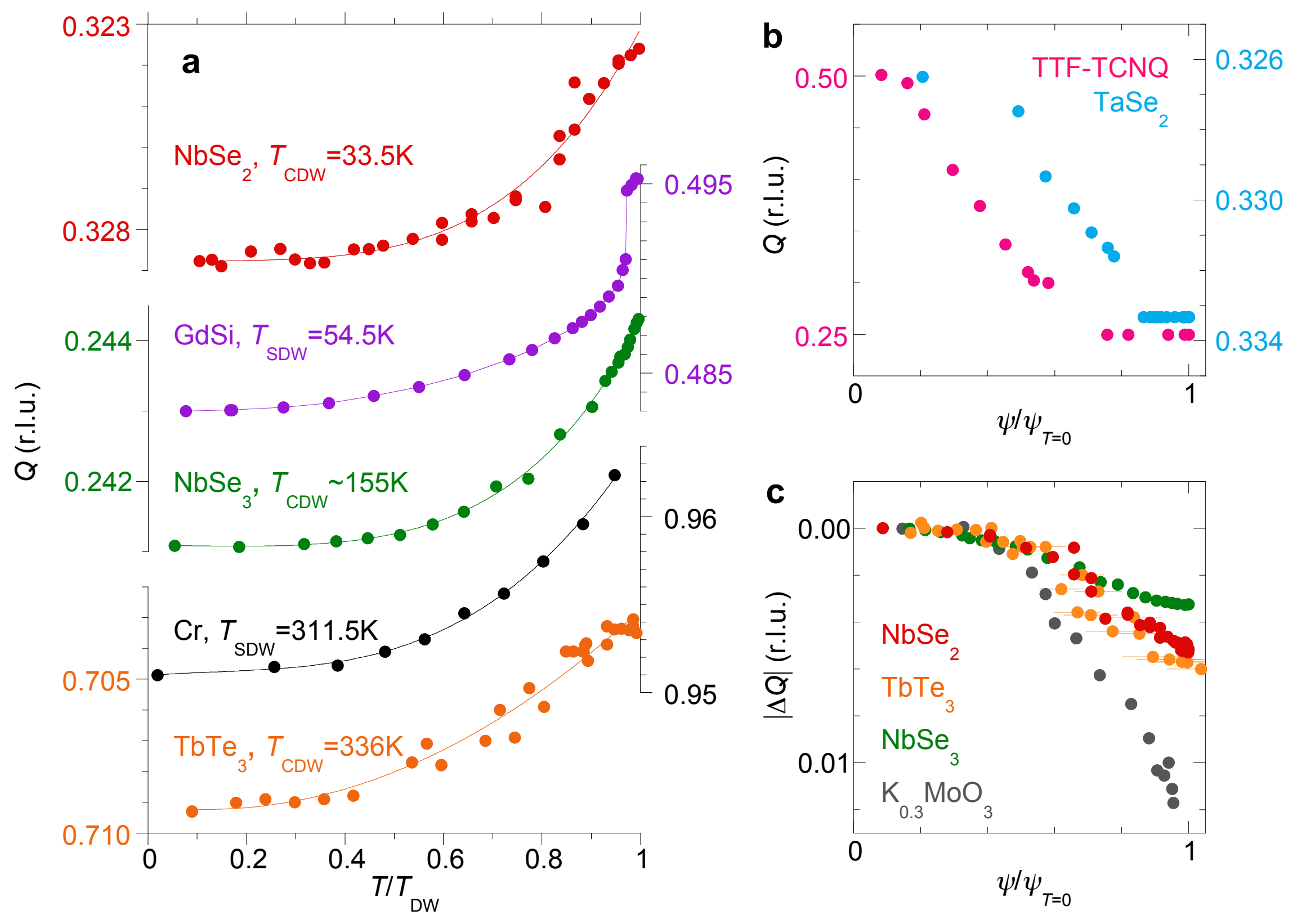


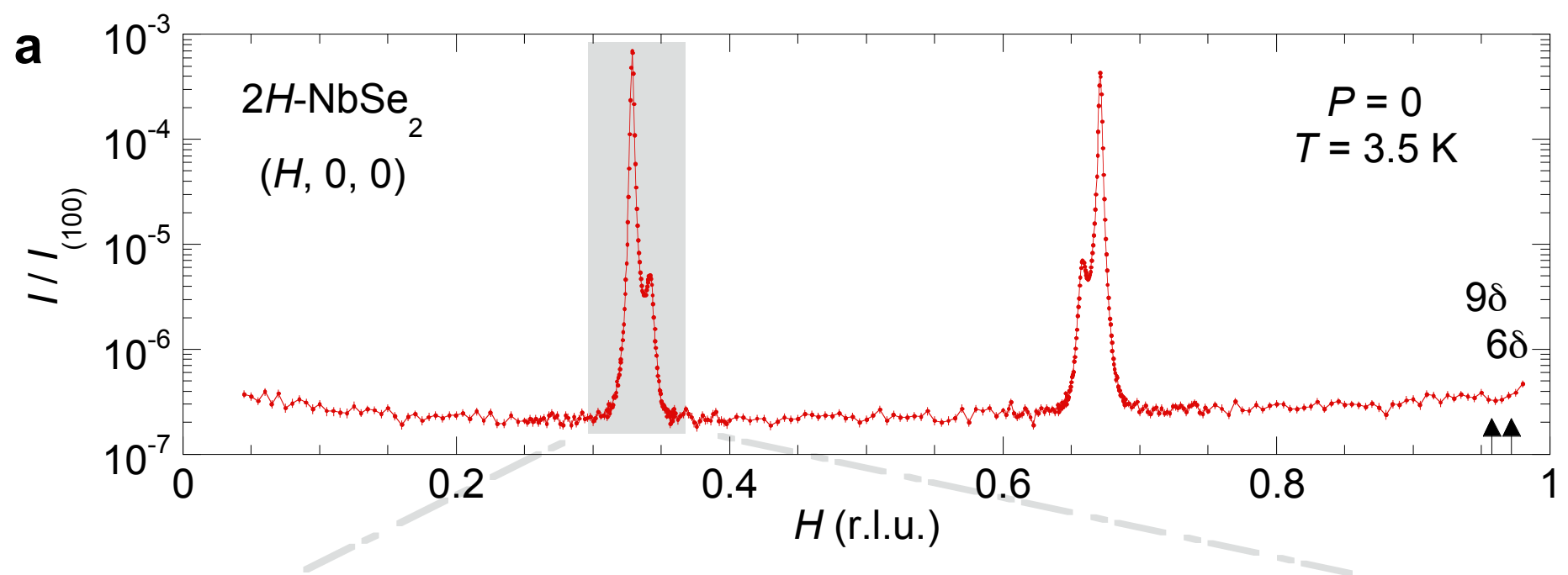

b

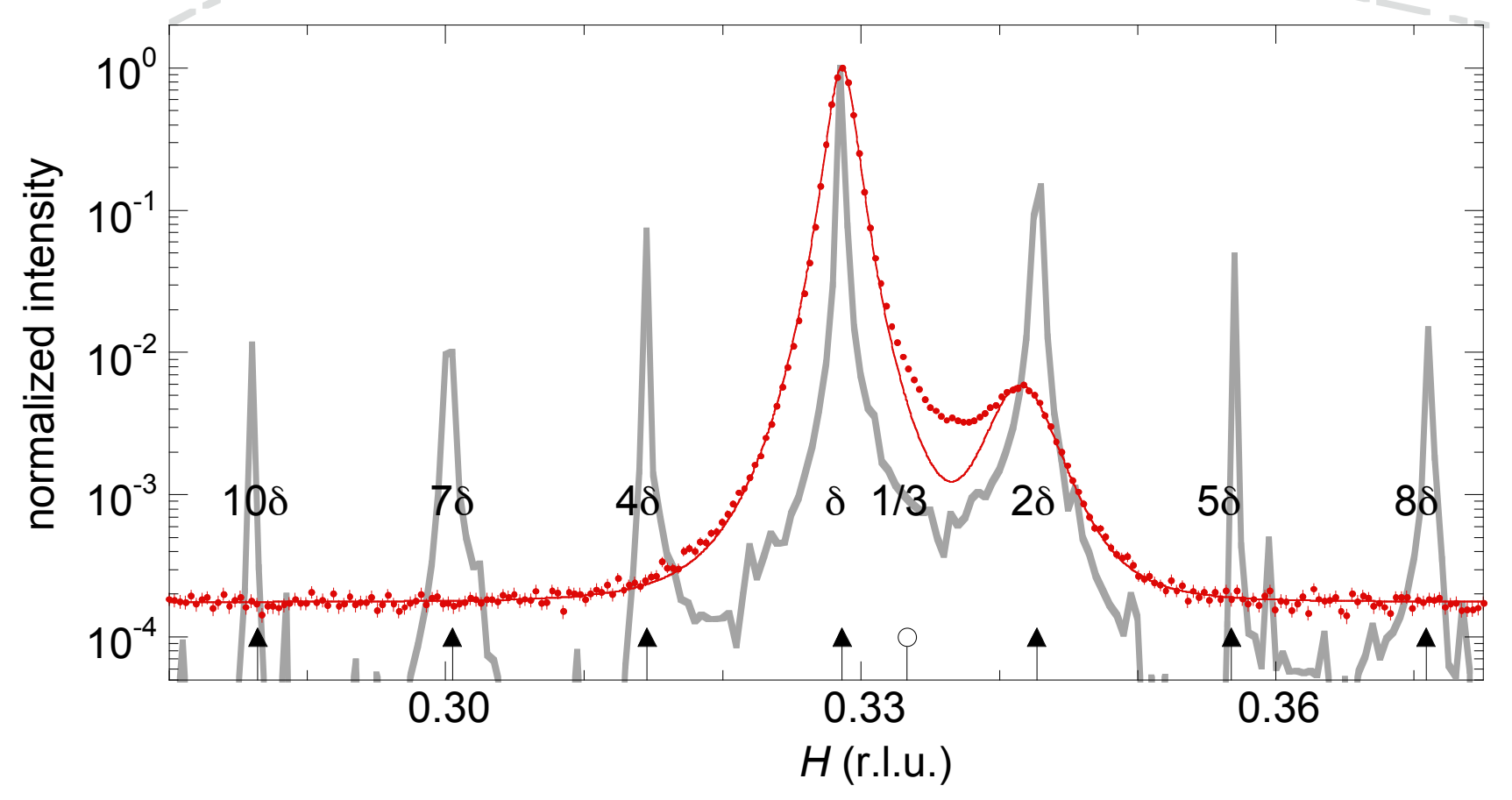




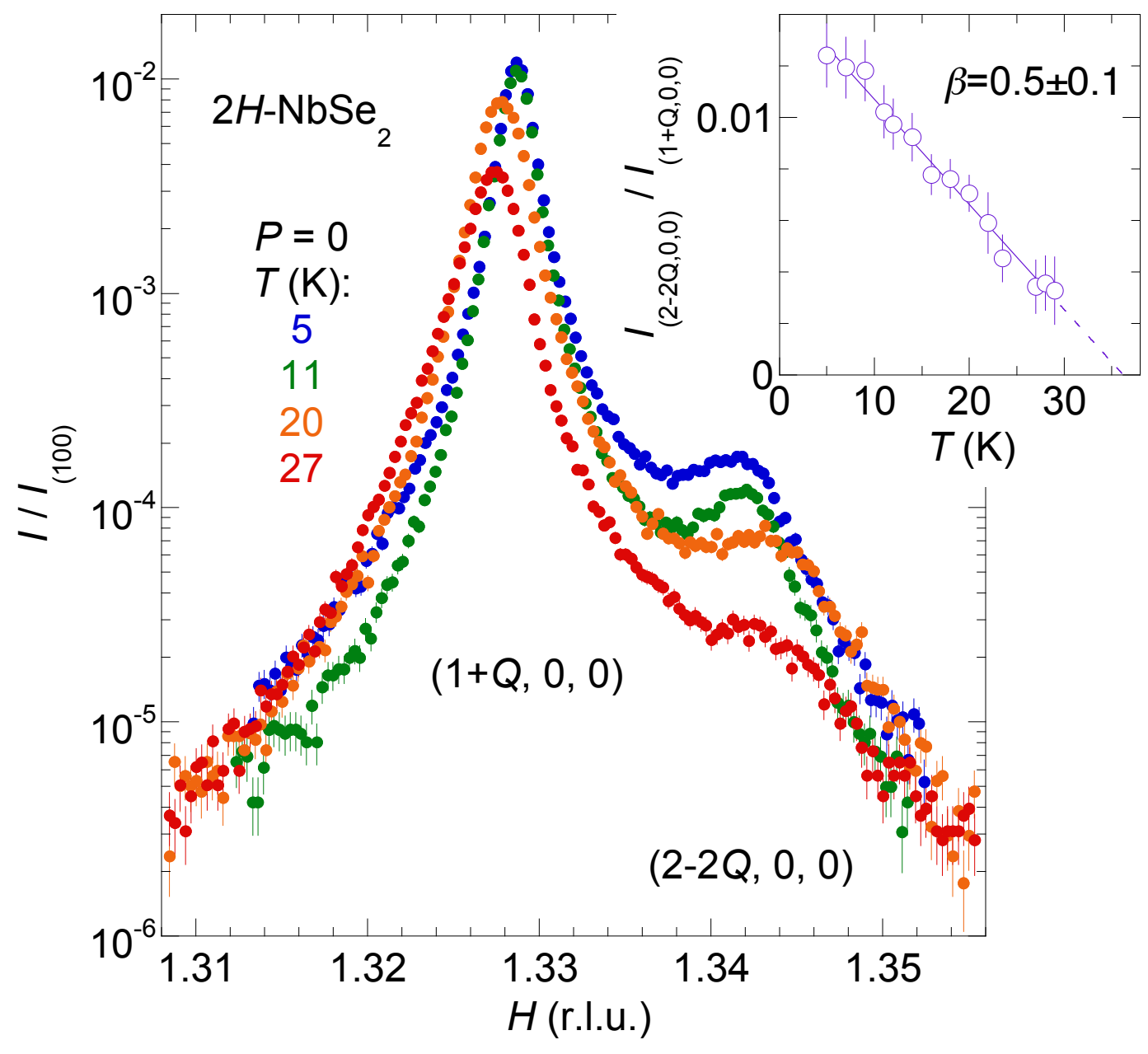




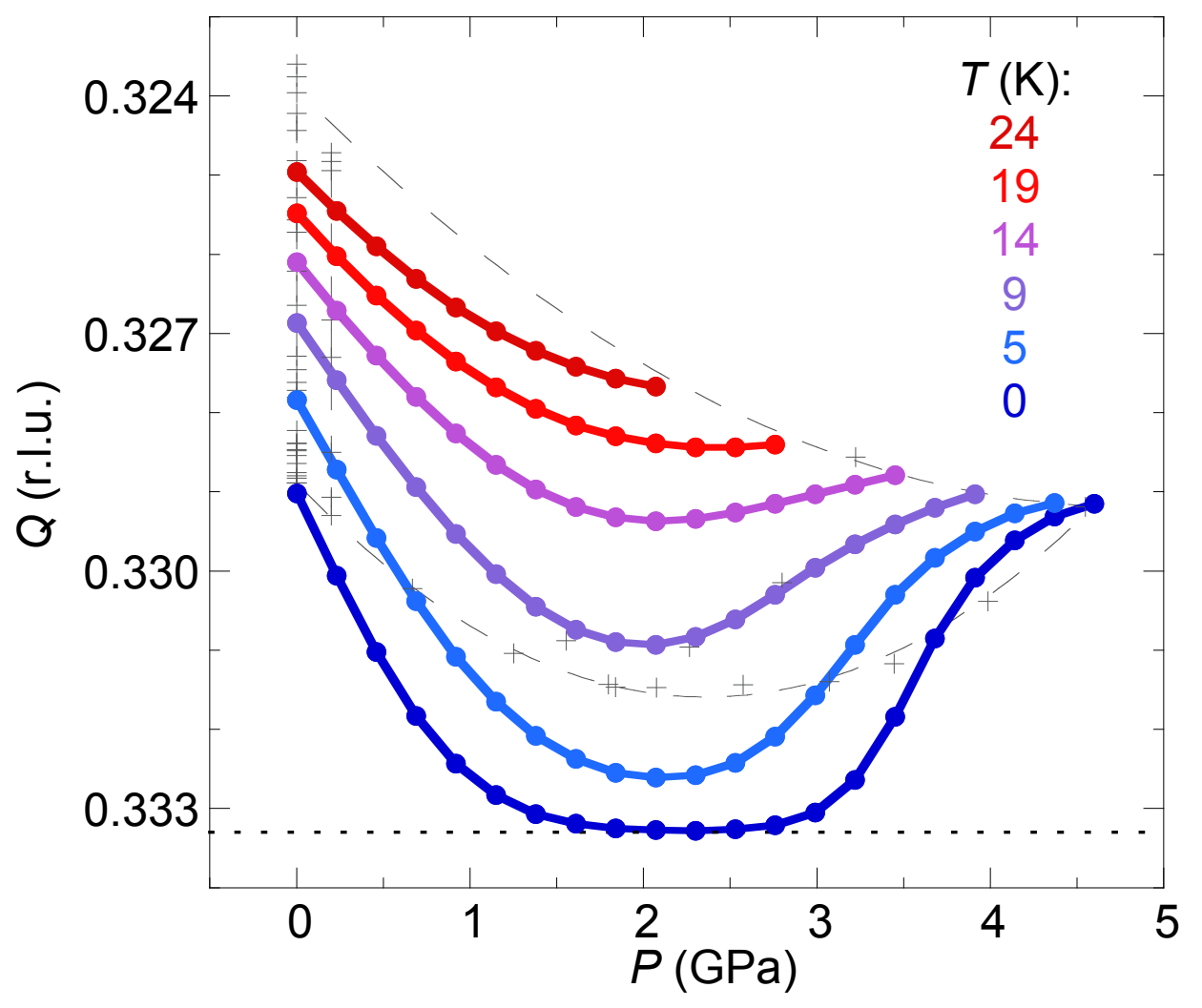




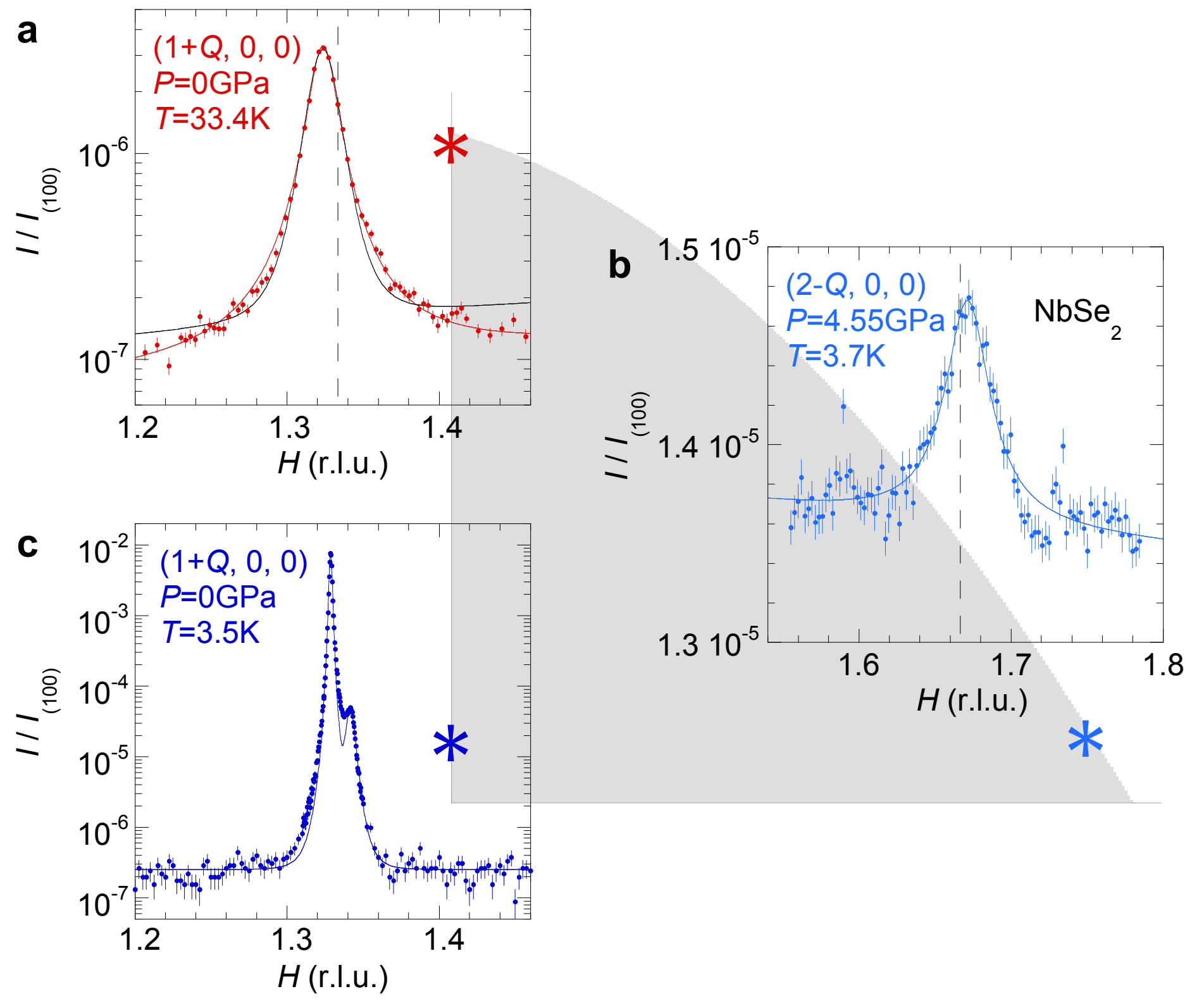

